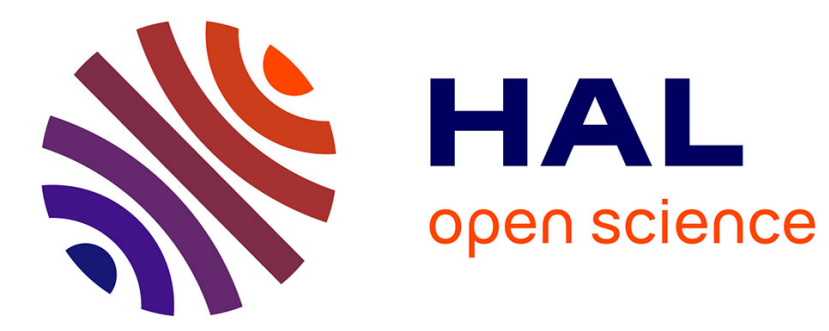

\title{
Contracting Under Unverifiable Monetary Costs
}

Nicolas Quérou, Antoine Soubeyran, Raphael Soubeyran

\section{To cite this version:}

Nicolas Quérou, Antoine Soubeyran, Raphael Soubeyran. Contracting Under Unverifiable Monetary Costs. Journal of Economics and Management Strategy, 2020, 29 (4), pp.892-909. 10.1111/jems.12389 . hal-02866383

\section{HAL Id: hal-02866383 \\ https://hal.inrae.fr/hal-02866383}

Submitted on 12 Jun 2020

HAL is a multi-disciplinary open access archive for the deposit and dissemination of scientific research documents, whether they are published or not. The documents may come from teaching and research institutions in France or abroad, or from public or private research centers.
L'archive ouverte pluridisciplinaire HAL, est destinée au dépôt et à la diffusion de documents scientifiques de niveau recherche, publiés ou non, émanant des établissements d'enseignement et de recherche français ou étrangers, des laboratoires publics ou privés.

\section{(이) $\$$}

Distributed under a Creative Commons Attribution - NonCommercial - NoDerivatives $\mid 4.0$ 


\title{
Contracting Under Unverifiable Monetary Costs*
}

\author{
Nicolas QuÉrou ${ }^{\dagger}$ Antoine Soubeyran ${ }^{\ddagger}$ Raphael Soubeyran ${ }^{\S}$
}

\begin{abstract}
We consider a contracting relationship where the agent's effort induces monetary costs, and limits on the agent's resource restrict his capability to exert effort. We show that, the principal finds it best to offer a sharing contract while providing the agent with an up-front financial transfer only when the monetary cost is neither too low nor too high. Thus, unlike in the limited liability literature, the principal might find it optimal to fund the agent. Moreover, both incentives and the amount of funding are non-monotonic functions of the monetary cost. These results suggest that an increase in the interest rate may affect the form of contracts differently, depending on the initial level of the former. Using the analysis, we provide and discuss several predictions and policy implications.
\end{abstract}

JEL classification: D82, D86.

Keywords: moral hazard, funding, wealth constraint, contract theory.

*Acknowledgements: We are grateful to Daniel Barron, Daniel Cardona, Ines Macho-Stadler, David PérezCastrillo, David Sappington, Julie Subervie, two referees, an Associate Editor and the Editor (Ramon CasadesusMasanell) for helpful comments and suggestions. This work was supported by the ANR GREEN-Econ research project (ANR-16-CE03-0005).

${ }^{\dagger}$ CEE-M, Univ. Montpellier, CNRS, INRAe, Institut SupAgro, Montpellier, France. Email: nicolas.querou@supagro.fr

${ }^{\ddagger}$ CNRS and EHESS, Aix-Marseille School of Economics, Aix-Marseille University, 13002 Marseille, France. E-mail: antoine.soubeyran@gmail.com

${ }^{\S}$ CEE-M, Univ. Montpellier, CNRS, INRAe, Institut SupAgro, Montpellier, France. Email: raphael.soubeyran@inrae.fr 


\section{Introduction}

Most of the literature on contracts assumes that the agent's effort cost is non-monetary, while there are many contracting relationships where this cost is at least partially pecuniary. For instance, this is the case for issues related to corporate social responsibility (Baron, 2007, Heyes and Martin, 2016) such as payment schemes for environmental services (see Alix-Garcia and Wolff, 2014). This is also consistent with cases where the agent is a profit-seeking company, and this agent's actions involve unverifiable monetary costs: Among other examples, subcontractors operating at arm's length face this type of costs, as in the automobile industry (Kawasaki and McMillan, 1987) or for construction projects. Even researchers working on a project funded by a public or private institution may declare spending more hours on this project than they actually do. In all these cases, the agent's effort implies non-monetary and monetary costs, which are often unverifiable due to high costs of monitoring or lack of expert knowledge.

The existence of monetary costs raises a number of general questions regarding the optimal contract between a principal and an agent. When is it in the principal's best interest to provide incentives or to fund an agent whose wealth is limited? What is the best incentive/funding structure to manage such a relationship? What is the effect of the magnitude of monetary costs on the specifics of the optimal contract? We attempt to answer these and related questions 1

In our environment the agent takes an unobserved costly action, which produces a stochastic output. The principal provides incentives by paying the agent based on the observed output and by providing him with, or by requiring, an up-front payment. Unlike in standard models, we consider that the agent's effort induces monetary costs (as well as non monetary costs, as in the standard setting). This cost can be covered by the agent's choice to undertake financial investments, while the principal can indirectly cover it through financial transfers to the agent. In this context, the agent's budget constraint restricts the set of feasible actions. Thus, the lower the level of wealth of the agent, including transfer from or to the principal, the lower the maximum effort level that the agent can provide. The principal and the agent are both risk neutral, so that the only distortion comes from the budget constraint, which limits the agent's set of feasible actions. When the budget constraint binds, the up-front payment and the effort become rivals. The agent cannot pay a large up-front payment and supply a high effort level.

We obtain several results that do not exist in the polar case of strictly non-monetary costs, even under limited liability, which is actually consistent with standard models used in Lewis and Sappington (2000a $\mathrm{b}, 2001)$. We first show that funding is sometimes optimal, but that it prevents the principal to get the full returns of the project. Second, the link between monetary costs and funding is not straightforward: funding is optimal if and only if the agent's wealth is sufficiently small and the monetary cost is neither too low nor too high. Indeed, when the monetary cost is small enough the agent can cover the full monetary cost of the project and, when this cost is sufficiently large, the principal has incentives to induce a decrease in the monetary costs through a decrease in the agent's share of profits, which allows the agent to also cover the monetary costs of the project.

Third, the optimal contract exhibits interesting features: the incentive part decreases as the

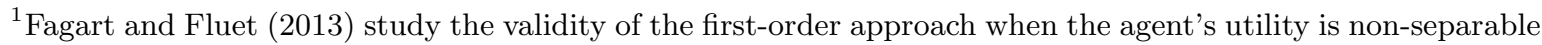
in the agent's income and effort cost. There is no wealth constraint in their setting. 
magnitude of the monetary costs increases up to a threshold value, and it then increases for larger values of these costs. Fourth, up to a threshold value of the monetary cost, the optimal bonus and the transfer both increase as the value of the project increases. When the monetary cost moves above this threshold, the bonus paid to the agent increases, while the transfer received from the agent decreases, as the project value increases. Fifth, we analyze welfare effects, and show that a larger monetary cost has a negative effect on the surplus of both parties.

We then discuss several predictions and policy implications. Our model predicts that payment for environmental services programs should not necessarily be more likely to fund projects when opportunity costs get larger. Moreover, an increase in the interest rate may notably affect the optimal contract design: depending on its initial level, an increase in the interest rate may (i) lead to an increase or a decrease in the strength of incentives (ii) increase or decrease the financial transfer (iii) affect the qualitative nature of the transfer (funding to or payment from the agent). The model also provides a rationale for the fact that outside financing and profit sharing be parts of the optimal contract when monetary costs are partially unobservable $2^{2}$

Finally, we discuss several extensions. We prove that our setting differs from the classical notion of limited liability, and we highlight the robustness of our results when the agent can borrow money from the principal, and when self-funding can be used as an outside option.

The present paper is related to the literature on moral hazard (Arrow, 1963, Pauly, 1968). The closest contributions are Lewis and Sappington (2000a b, 2001), where the focus is on how constraints on the agent's wealth impede on the efficient allocation of resources by limiting the optimal tailoring of the reward structure 3 In these contributions, the agent's effort does not induce any monetary cost, and we obtain novel results: we prove the optimality of funding, show that funding occurs for intermediate values of the monetary cost, highlight the non-monotonic effect of this cost on incentives, and that funding and incentives may be complementary.

This work also highlights the optimal trade-off between funding and incentives 4 Unlike most of the literature on financial contracts (see Bester, 1987 and Innes, 1990), we consider that the investment level is endogenous. We thus contribute to the important question of optimal contracting when both investment policy choices and effort are made privately by the agent.

The effect of monetary costs induced by the agent's effort is conceptually different from the notion of limited liability (see Macho-Stadler and Pérez-Castrillo, 2018, and Sappington, 1983) 5 Limited liability might either bound the feasible payments from the principal to the agent (which must lie above an exogenous threshold, see Jewitt et al., 2008, and Poblete and Spulber, 2012) or the agent's ex-post payoff. We show this conceptual difference in Section $5^{6}$

The remainder of the paper proceeds as follows. The model is provided in Section 2, and the analysis in Section 3 . (Policy) implications are discussed in Section 4 . Section 5 discusses some of the assumptions. Finally, Section 6 concludes. All proofs are provided in an appendix.

\footnotetext{
${ }^{2}$ Here profit sharing is equivalent to a share of proceeds in case the project is successful.

${ }^{3}$ Agents are assumed to make bond payments at the outset of the relationship, even though the principal can promise to return some portion of the initial bond in Lewis and Sappington (2001).

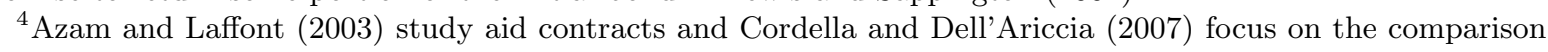
of budget support and project aid. These articles do not study the optimal mix.

${ }^{5}$ See also Ohlendorf and Schmitz (2012) or Chassang (2013) for recent contributions.

6 Laffont and Matoussi (1995) consider a moral hazard problem in which the agent has limited budget for a verifiable input. There is no potential relationship between wealth and effort level. Che and Gale (2000) study selling mechanisms with limited budget under adverse selection and Burkett (2015) consider the case with budget manipulation by the agent (see also Benoît and Krishna, 2001).
} 


\section{The Model}

Consider a principal and an agent who may enter into a relationship to develop a project. The outcome of the project can be either a success or a failure. If successful, the relationship yields returns $V>0$, while failure results in zero return. The agent's effort $e, e \in[0,1]$, determines the probability of success $p(e)=e$ of the relationship. The agent incurs both a monetary cost $K(e)=\theta \frac{e^{2}}{2}$, with $\theta \geq 0$ and a non monetary cost $D(e)=\psi \frac{e^{2}}{2}$ with $\psi \geq 0$ when exerting effort $e \geq 0$. He has a limited budget $B \geq 0$. The principal is the residual claimant of the output.

The contractual relationship between the principal and the agent has several features. The agent's effort is not verifiable, thus the contract is only based on the success or failure of the project. It specifies both an up-front payment from the principal to the agent, $T$ (which may be negative or positive), and a bonus paid to the agent, $w$, only in case of success. If the agent rejects the contract he consumes his wealth, $B$. If he accepts the contract, the principal "pays" him the up-front payment $T$, which is possibly a payment from the agent to the principal $[7$ The principal's problem is thus as follows 8

$$
\underset{T, w, e}{\operatorname{Max}} E u^{P}=e(V-w)-T
$$

such that the following conditions hold:

$$
\begin{gathered}
e=\underset{\left\{e \geq 0 \mid B+T \geq \theta \frac{e^{2}}{2}\right\}}{\arg \max }\left\{e w+B+T-\psi \frac{e^{2}}{2}-\theta \frac{e^{2}}{2}\right\}, \\
E u^{A}=e w+B+T-\psi \frac{e^{2}}{2}-\theta \frac{e^{2}}{2} \geq B,
\end{gathered}
$$

Expression (P0) reflects the fact that the principal's expected net return is the difference between the expected surplus from the project and the rent that accrues to the agent, that is, the bonus $w$ and the up-front payment, $T$. Condition ( $\mathrm{PC} 0)$ corresponds to the participation constraint.

Condition $\mathrm{IC0}$ corresponds to the incentive constraint, which ensures that the effort level provides the maximal payoff to the agent. This incentive constraint is novel as it includes a budget constraint, $B+T \geq \theta \frac{e^{2}}{2}$. Specifically, it guarantees that the agent cannot bear a monetary cost of effort that exceeds the sum of his budget and the up-front payment. When $\theta=0$, exerting effort induces a purely non-monetary cost, the agent's effort level and the upfront payment received from the principal are not linked through the budget constraint, which corresponds to $B+T \geq 0$ here. This is entirely similar to the problem analyzed in Lewis and Sappington (2000a b, 2001). By contrast, when $\theta>0$, exerting effort induces a monetary cost: the agent's effort level and the up-front payment received from the principal are linked through the budget constraint $B+T \geq \theta \frac{e^{2}}{2}$. The agent's effort is then bounded above by $\sqrt{\frac{2(B+T)}{\theta}}$. This upper bound increases as the agent's budget increases, as the transfer the agent receives from the principal increases, and as the monetary $\operatorname{cost} \theta$ decreases 9 A focus will be put on how the

\footnotetext{
${ }^{7} \mathrm{As}$ in a notable part of the literature we consider that the parties' outside options yield zero return. We will discuss the robustness of our results when outside options yield positive returns in Section 5

$\varepsilon$ Jelovac and Kembou-Nzale 2020) consider a similar type of problem in a joint production setting, where a contract specifies a transfer from the principal to the agent and the principal's input in the joint production.

${ }^{9}$ Unlike most models of financial contracts, which consider adverse selection problems and focus on issues of risk sharing, the focus is on problems raised by potentially endogenous monetary investments in a moral hazard
} 
monetary cost affects the optimal contract compared to the benchmark case where $\theta=0$.

\section{Analysis}

We first characterize the first-best situation, as this will be the benchmark for the analysis.

\subsection{The First-best Situation}

The first best effort level maximizes the joint payoff of the agent and the principal:

$$
\underset{e \geq 0}{\operatorname{Max}} J=e V-\psi \frac{e^{2}}{2}-\theta \frac{e^{2}}{2}
$$

The interior solution is given by

$$
e^{F B}=\frac{V}{\psi+\theta}
$$

Effort level $e^{F B}$ increases in project value $V$, and decreases in $\operatorname{costs} \theta$ and $\psi$.

\subsection{A Simplified Problem}

Before characterizing the solution to problem $(\overline{\mathrm{P} 0})$, let us introduce the simpler optimization problem $(\mathrm{P})$ :

$$
\underset{T, w, e}{M a x} E u^{P}=e(V-w)-T
$$

such that the following conditions hold:

$$
\begin{gathered}
e=\frac{w}{\psi+\theta}, \\
E u^{A}=e w+B+T-\psi \frac{e^{2}}{2}-\theta \frac{e^{2}}{2} \geq B, \\
B+T \geq \theta \frac{e^{2}}{2} .
\end{gathered}
$$

The difference with initial problem $\mathrm{P} 0$ is that the budget constraint $B+T \geq \theta \frac{e^{2}}{2}$ is considered independently from the incentive constraint. Now, we proceed in two steps to characterize the solution to problem $(\mathrm{P} 0)$. First, when the budget is large enough, we show that the principal induces the first-best outcome by using one out of two feasible contracts. Second, when the budget is not too large, we show that the solution to $(\mathrm{P} 0)$ is the same as that of problem $(\overline{\mathrm{P}})$. We can then focus on this simplified setting to characterize the solution (see Appendix B) 10

\subsection{Optimal Management: Motivating versus Funding}

We now assume that the effort is non contractible, and the agent faces budget constraint (BC).

setting. A moral hazard model of financial contracts is provided in Section 6 in Bester (1987), but it assumes perfect competition between principals, risk-averse agents, and exogenous monetary investment.

${ }^{10}$ The approach follows a similar idea than the first-order approach (Mirrlees, 1976, Rogerson, 1985, Kirkegaard, 2017), with the difference that there is a new constraint on the agent's side that must be accounted for. 


\subsubsection{No Funding in Polar Cases}

When the agent's budget is large enough (above $B_{2}(\theta)$, that we define below), the principal induces the first-best outcome by relying on one of the two following options ${ }^{11}$ First, the agent could be made residual claimant for all returns from the project $(w=V)$, then the effort level is first best $\left(e=\frac{V}{\psi+\theta}\right)$ and the principal receives an up-front payment from the agent equal to the expected surplus $\left(T=-J^{F B}=-\frac{1}{2} \frac{V^{2}}{\psi+\theta}<0\right)$. In this case, the budget constraint is not binding. Second, the principal could specify $w=\left(\frac{\psi+\theta}{V}\right) B+\frac{\psi}{2}\left(\frac{V}{\psi+\theta}\right)$ and require a transfer $T=\frac{\theta}{2}\left(\frac{V}{\psi+\theta}\right)^{2}-B{ }^{12}$ In this case the budget constraint is binding. Both options lead to the same effort and the same payoffs for both parties. The budget constraint must be such that the wealth of the agent is larger than the expected surplus gross of monetary cost:

$$
B \geq J^{F B}+\theta \frac{\left(e^{F B}\right)^{2}}{2}=e^{F B} V-\psi \frac{\left(e^{F B}\right)^{2}}{2} .
$$

Let $B_{2}(\theta)$ denote the right hand side in (3). It can be written as follows:

$$
B_{2}(\theta)=\frac{\psi+2 \theta}{2}\left(\frac{V}{\psi+\theta}\right)^{2} \text {. }
$$

Condition (3) holds when $V$ is sufficiently low and the cost parameters are sufficiently large. Intuitively, the expected surplus gross of monetary cost $G=J+\theta \frac{(e)^{2}}{2}$ decreases when the value of the project decreases and when the non monetary cost parameter $\psi$ increases. Moreover, since the agent bears the monetary cost, the optimal effort level $\left(e^{F B}=\frac{V}{\psi+\theta}\right)$ is lower than the effort that maximizes the expected surplus gross of monetary cost $\left(e=\frac{V}{\psi}\right)$. Thus, the expected surplus gross of monetary cost $G$ increases when $e$ increases at $e=e^{F B}$. Since the first best effort level $e^{F B}$ decreases when $\theta$ increases, $G$ decreases when $\theta$ increases. So, when the monetary cost is sufficiently large, the principal can implement the first best and there is no funding $T<0$. Thus, we obtain:

Proposition 1: If the agent's budget is large enough $\left(B \geq B_{2}(\theta)\right)$ or, equivalently, if the monetary cost is large enough $\left(\theta \geq 0\right.$ if $B \geq \frac{V^{2}}{2 \psi}$ and $\theta \geq \theta_{2} \equiv \frac{V^{2}-2 \psi B+V \sqrt{V^{2}-2 \psi B}}{2 B}$ if $\left.B \leq \frac{V^{2}}{2 \psi}\right)$, the optimal up front payment is a transfer from the agent to the principal, $T^{*} \leq 0$.

Hence, there is no funding when the monetary cost parameter is large enough. Combining this result with the fact that the no-funding property also holds when $\theta=0$ (Lewis and Sappington (2000a) $){ }^{13}$ we can make the following observation:

Remark: There is no funding in the polar cases where $\theta=0$ or where $\theta$ is large enough.

In Subsection 3.3.2, we will show that no funding may also hold when $\theta$ is small.

\footnotetext{
${ }^{11}$ See Appendix B.

${ }^{12}$ This option is optimal provided $B>B_{2}(\theta)$ so $T \leq 0$ is satisfied.

${ }^{13}$ Indeed, when $B<B_{2}(\theta)$, we have $T=\theta \frac{e^{2}}{2}-B$. Hence, if $\theta=0$ we have $T=-B<0$.
} 


\subsubsection{Funding}

We now assume that $B<B_{2}(\theta)$ holds. In this case, the budget constraint is necessarily binding, $T=\theta \frac{e^{2}}{2}-B$, and the transfer from the principal to the agent covers the monetary cost net of the agent's wealth (see Appendix B). Notice that the incentive constraint can be replaced by $e=\frac{w}{\psi+\theta}$. The principal's program can be rewritten as follows:

$$
\underset{e, w}{\operatorname{Max}} E u^{P}=e V-\frac{\psi+\theta}{2} e^{2}-\left[e w-\frac{\psi}{2} e^{2}-B\right]
$$

such that the following conditions hold:

$$
\begin{gathered}
e=\frac{w}{\psi+\theta}, \\
E u^{A}=e w-\frac{\psi}{2} e^{2} \geq B .
\end{gathered}
$$

The principal will receive a transfer from the agent if $\theta \frac{e^{2}}{2} \leq B$ holds. He will provide funding if the monetary cost is larger than the agent's wealth, $\theta \frac{e^{2}}{2}>B$. Compared to the first best situation, the principal has incentives to choose a low bonus $w$ to induce a low effort level and in turn a low monetary cost, $e^{*}<e^{F B}$. We now define the following thresholds:

$$
B_{1}(\theta)=\frac{\psi+2 \theta}{2}\left(\frac{V}{2 \psi+3 \theta}\right)^{2}<B_{2}(\theta) ; \quad B_{0}(\theta)=\frac{\theta}{2}\left(\frac{V}{2 \psi+3 \theta}\right)^{2}<B_{1}(\theta) .
$$

The basic properties of the optimal contract are as follows:

Lemma 1: When $B<B_{2}(\theta)$ holds, the optimal contract exhibits the following properties:

(i) Inefficiency: the effort exerted by the agent is strictly lower than the first best effort level, $0<e^{*}<e^{F B}$.

(ii) Shared returns: the optimal contract specifies the share of profits $w^{*}=\frac{\psi+\theta}{2 \psi+3 \theta} V<V$ when $0 \leq B<B_{1}(\theta)$ holds and $w^{*}=(\psi+\theta) \sqrt{\frac{2 B}{\psi+2 \theta}}<V$ when $B_{1}(\theta) \leq B<B_{2}(\theta)$ is satisfied.

(iii) Rents: the agent gets a strictly positive surplus when $0 \leq B<B_{1}(\theta)$ while he does not get any rent when $B_{1}(\theta) \leq B<B_{2}(\theta)$ is satisfied.

(iv) Funding: the up-front transfer is a payment from the principal to the agent $\left(T^{*}>0\right)$ only when $0 \leq B<B_{0}(\theta)$.

When the agent exerts the first-best effort level, the principal cannot ask for an up-front payment larger than $T=\frac{\theta}{2}\left(e^{F B}\right)^{2}-B<0$, and the agent gets a large rent, which is not optimal. When $B_{1}(\theta) \leq B<B_{2}(\theta)$ the optimal values of the bonus and of the transfer are fully characterized by the binding participation and budget constraints ${ }^{14}$ This explains why the agent gets no rent unlike in the first sub-case. Notice that $B_{1}(\theta) \leq B$ ensures that the agent's participation constraint is binding, and $B<B_{2}(\theta)$ ensures that the budget constraint is binding. Due to the discussion provided in Lemma 1 , the most important result is that funding may be optimal (i.e. $T^{*}>0$ ) when $0 \leq B<B_{0}(\theta){ }^{15}$ We now characterize the situations in

\footnotetext{
${ }^{14}$ When $0 \leq B<B_{1}(\theta)$ holds the incentive part of the contract is increasing in the value of the project, consistently with Holmstrom (1979).

${ }^{15}$ We show that this conclusion is not consistent with the classical notion of limited liability in Section 5.
} 
which this occurs. To do so, we first discuss how the optimal bonus is affected by a change in the monetary cost.

Proposition 2: There exists a threshold $\theta_{1}$ satisfying $0 \leq \theta_{1} \leq \theta_{2}$ and such that, when the monetary cost $\theta$ increases, the optimal bonus $w^{*}$ decreases for $\theta<\theta_{1}$ and increases for $\theta_{1}<\theta \leq \theta_{2} \cdot{ }^{16}$

Figure 1: Monetary cost and incentives



If constraint $\left(\mathrm{PC}^{\prime}\right)$ is not binding, the optimal bonus is $w^{*}=\frac{\psi+\theta}{2 \psi+3 \theta} V$, which decreases when $\theta$ increases. Indeed, the budget constraint $(\overline{\mathrm{BC}})$ is binding and the principal has to bear the exceeding monetary cost $\left(T=\theta \frac{e^{2}}{2}-B\right)$, thus he will choose to induce a lower effort level -using a lower bonus- when the monetary cost is larger.

Using the incentive constraint $\mathrm{IC}^{\prime}$, constraint $\mathrm{PC}^{\prime}$, can be written as $w \geq \underline{w}=(\psi+\theta) \sqrt{\frac{2 B}{\psi+2 \theta}}$. Thus, the bonus is bounded below, and the lower bound increases when $\theta$ increases. This result is not due to the quadratic specification. Indeed, the incentive constraint characterizes the effort level as a function of $(w, \theta), e(w, \theta)=\frac{w}{w+\theta}$. The lower bound $\underline{w}$ is such that the participation constraint is binding, $E u^{A}(w, e(w, \theta))=B$. It then suffices to highlight that $E u^{A}(w, e(w, \theta))$ increases when the bonus increases, and that it decreases when the monetary cost increases.

Regarding the effect of the bonus, we know that $e=\frac{w}{\psi+\theta}$, which is lower than $e=\frac{w}{\psi}$ that maximizes the agent's expected surplus $\left(E u^{A}=e w-\frac{\psi}{2} e^{2}\right)$. Since this expected surplus is increasing for $e$ up to $\frac{w}{\psi}$, this expected surplus increases when $e$ increases as long as the incentive constraint holds. As a consequence, the indirect effect $\frac{\partial E u^{A}}{\partial e} \frac{\partial e}{\partial w}$ is positive. Since the direct effect $\frac{\partial E u^{A}}{\partial w}$ is also positive, the overall effect is positive. Finally, it is easily checked that $E u^{A}(w, e(w, \theta))$ decreases when the monetary cost $\theta$ increases. Indeed, an increase in $\theta$ affects the agent's expected surplus only through the effort level, and this indirect effect $\frac{\partial E u^{A}}{\partial e} \frac{\partial e}{\partial \theta}$ is negative. We also deduce that the participation constraint is not binding when the monetary cost $\theta$ is sufficiently low $\left(\theta<\theta_{1}\right)$ and it is binding otherwise $\left(\theta_{1}<\theta \leq \theta_{2}\right){ }^{17}$ Then, using $e^{*}=\frac{w^{*}}{\psi+\theta}$ and Proposition 2, when the budget constraint is binding while the participation constraint is not binding $\left(\theta<\theta_{1}\right)$, the effort level decreases when $\theta$ increases. When both the budget and the participation constraints are binding $\left(\theta_{1}<\theta \leq \theta_{2}\right)$, we know that the agent's expected surplus increases when the effort level increases. Moreover, when the participation constraint is binding, the optimal bonus increases when $\theta$ increases. All together, the optimal

\footnotetext{
${ }^{16}$ Formally, $B<B_{1}(\theta)$ and $\theta \geq 0 \Leftrightarrow 0 \leq \theta<\theta_{1}$ and $B<\frac{V^{2}}{8 \psi}$, where $\theta_{1}=\frac{V^{2}-12 \psi B+V \sqrt{V^{2}-6 \psi B}}{18 B}$.

${ }^{17}$ Recall that the case in which the monetary cost is larger than $\theta_{2}$ has been studied in the previous section. In this case, the participation constraint is binding while the budget constraint is not.
} 
effort decreases when $\theta$ increases, so that the participation constraint remains binding. Thus, the effect of a larger $\theta$ on $T^{*}=\theta \frac{\left(e^{*}\right)^{2}}{2}-B$ is non-monotonic. We obtain:

Proposition 3: When $B<B_{2}(\theta)$ holds, funding is optimal (i.e. $T^{*}>0$ ) if and only if the agent's wealth is sufficiently small and the monetary cost is neither too low nor too high: $B<\frac{V^{2}}{48 \psi}$ and

$$
\frac{V^{2}-24 B \psi-V \sqrt{V^{2}-48 B \psi}}{36 B}<\theta<\frac{V^{2}-24 B \psi+V \sqrt{V^{2}-48 B \psi}}{36 B} .
$$

When the monetary cost parameter is small enough, the agent can cover the full monetary cost of the project. When the monetary cost parameter is sufficiently large, the principal has sufficiently strong incentives to decrease the monetary cost through a decrease in the agent's share of profits, which allows him to cover the full monetary cost of the project $\left(B \geq \theta \frac{\left(e^{*}\right)^{2}}{2}\right)$. When the monetary cost parameter value is neither too low nor too high, the principal's incentives to decrease the monetary cost of the project are not as strong, and he will provide funds (see Figure 2).

Figure 2: Monetary cost and funding



Before moving on to the next subsection, we finally characterize the cases where funding or no funding occurs. Indeed $\frac{V^{2}-24 B \psi-V \sqrt{V^{2}-48 B \psi}}{36 B}$ is positive whenever $B<\frac{V^{2}}{48 \psi}$ is satisfied. Moreover $\frac{V^{2}-24 B \psi+V \sqrt{V^{2}-48 B \psi}}{36 B}<\theta_{1}$ holds as this is equivalent to $V^{2}+2 V \sqrt{V^{2}-6 \psi B}>V \sqrt{V^{2}-48 \psi B}$ which always holds whenever $B \leq \frac{V^{2}}{48 \psi}$ is satisfied. All together, we have:

$$
0<\frac{V^{2}-24 B \psi-V \sqrt{V^{2}-48 B \psi}}{36 B}<\frac{V^{2}-24 B \psi+V \sqrt{V^{2}-48 B \psi}}{36 B}<\theta_{1}<\theta_{2}
$$

Combining Propositions 1 and 3, we thus conclude that no funding occurs when: the budget is large enough $\left(B \geq \frac{V^{2}}{48 \psi}\right)$; the budget and the monetary cost are small enough $\left(B<\frac{V^{2}}{48 \psi}\right.$ and $\left.\theta \leq \frac{V^{2}-24 B \psi-V \sqrt{V^{2}-48 B \psi}}{36 B}\right)$; the budget is small enough and the monetary cost is sufficiently large $\left(B<\frac{V^{2}}{48 \psi}\right.$ and $\left.\theta \geq \frac{V^{2}-24 B \psi+V \sqrt{V^{2}-48 B \psi}}{36 B}\right)$.

\subsection{The effect of the project value}

Given that the optimal contract stipulates, in some cases, an up-front payment to the agent and a bonus payment, we analyze the effect of the project value on the optimal contract.

Proposition 4: (i) If $\theta \geq \theta_{2}$, optimal incentives and transfer to the agent are "substitutes" as regards the value of the project: incentives increase, and transfer decreases, as this value 
increases. Formally, $\frac{\partial w^{F B}}{\partial V}>0$ and $\frac{\partial T^{F B}}{\partial V}<0$.

(ii) If $\theta<\theta_{2}$, optimal incentives and transfer to the agent are "complementary" as regards the value of the project: as this value increases, the incentives and the transfer increase. Formally, $\frac{\partial w^{*}}{\partial V} \geq 0$ and $\frac{\partial T^{*}}{\partial V} \geq 0$.

When the budget constraint is binding, both the agent's share of profits and the transfer from the principal to the agent increases with the value of the project. When the value of the project increases, the marginal value of effort increases. Thus the principal induces a larger effort from the agent which in turn generates a larger monetary cost. Since the agent's budget constraint is binding, the principal has to make a larger transfer to the agent.

\subsection{Welfare Effects}

We now analyze how changes in the magnitude of monetary costs affect the surplus distribution.

Proposition 5: A larger $\theta$ has a negative effect on the surplus of both parties.

When $\theta_{2} \leq \theta$ the principal gets the full surplus $E u^{P}=J^{F B}=\frac{1}{2} \frac{V^{2}}{\psi+\theta}$, which decreases when $\theta$ increases. When $\theta \leq \theta_{2}$, the principal's surplus decreases when $\theta$ increases, and this property is fairly general. Indeed, this expected surplus can be defined as $E u^{P}(w, e(w, \theta), \theta)=e(V-w)-\theta \frac{e^{2}}{2}$ where $e(w, \theta)=\frac{w}{\psi+\theta}$. Hence, the optimal bonus $w^{*}$ is such that the optimality condition $\frac{\partial E u^{P}}{\partial w}+\frac{\partial E u^{P}}{\partial e} \frac{\partial e}{\partial w}=0$ is satisfied. We moreover know that $\frac{\partial e}{\partial w}>0$ and $\frac{\partial E u^{P}}{\partial w}<0$, and then $\frac{\partial E u^{P}}{\partial e}>0$. Thus, an increase in $\theta$ has a direct effect and an indirect effect (through the effect of $\theta$ on the effort level) on the principal's expected surplus, which are both negative.

When $\theta_{2} \leq \theta$ or $\theta_{1}<\theta \leq \theta_{2}$, the agent gets no rent. When $\theta<\theta_{1}$ the agent's expected surplus decreases when $\theta$ increases, and this property is fairly general. Indeed, an increase in $\theta$ has two indirect effects on expected surplus $E u^{A}\left(w^{*}, e^{*}\right)=e^{*} w^{*}-\psi \frac{\left(e^{*}\right)^{2}}{2}$. The first indirect effect goes through $e^{*}$ and we know that it is negative. The second indirect effect goes through $w^{*}$, and is given by $\frac{\partial E u^{A}}{\partial w^{*}} \frac{\partial w^{*}}{\partial \theta}$ which, using Proposition 2, is known to be negative. All together, the agent's expected surplus decreases when $\theta$ increases.

\section{Policy Implications}

The implications of the analysis apply generically to situations where the agent's action involves unverifiable monetary costs, which is the case for many important settings. We first notice that our model provides implications for the emergence of outside financing. Another empirical implication is that outside financing is more frequent when the interest rate is neither too low nor too high. Now, we choose to describe most of the implications of the analysis in the context of the design of payments for environmental service ${ }^{18}$ for expositional simplicity, and because this is getting increasing attention in discussions related to corporate social responsibility.

Payments for Environmental Services (PES) are contracts stipulating conditional payments to resource owners, which are effective if pre-defined conservation targets are achieved. Our model can be reinterpreted in the context of PES as follows. A forest owner (the agent) contracts

\footnotetext{
${ }^{18}$ See Alix-Garcia and Wolff $(2014)$ for a survey.
} 
with a private company or a Non-Governmental Organization (the principal) on a given share of land over a given period of time 19 The organization values the plot of land, and the value of the project corresponds here to the organization benefit if the plot of forest is conserved during the period of time (its value is zero if the plot of forest is not conserved). Ex-ante, there is a given number of trees on this land, which is (imperfectly) measured thanks to satellite imagery or to an expert visual evaluation. The contract stipulates that the forest owner receives an up-front payment and gets an additional payment if the plot of land under contract is detected as conserved at the end of the period 20 Some trees can be removed because of accidental fire or unexpected tree disease, and some trees can also be cut by the forest owner ${ }^{21}$ It may be difficult and costly to assess whether trees were destroyed because of natural hazard or because of the owner's will. The owner chooses the number of trees that he cuts during the given period of time, and the remaining trees are conserved: he thus chooses the magnitude of his conservation effort. The owner gets a benefit from cutting trees, and must get incentives to conserve any number of them. The probability that the plot of land is declared to be "conserved" (thus, that the outcome of the project is successful) increases with the number of trees conserved. A typical owner is often financially constrained: he has a limited level of wealth, but needs to use some amount of money in order to live during the period covered by the contract. The owner may also need to switch to other economic activities (e.g. intensive farming), which requires buying capital (production technologies) and/or acquiring specific knowledge related to these economic activities. If the owner does not accept the contract, he cuts the forest and consumes his wealth.

Within this setting we now discuss the issue of effective program design. Indeed, since forest owners face monetary opportunity costs, their level of wealth is constrained and may restrict the range of conservation actions they can undertake. As explained in Jayachandran (2013), a major drawback is that this instrument does not take into account the short-term large opportunity costs that liquidity constrained forest owners may face. However, Alix-Garcia and Wolff (2014) argue that contracts vary from 5 years in Mexico to 20 years in Ecuador, and that PES programs tend to pay at the end of each contract year and not at the end of the contract. In the present setting, there is a possibility that the funding organization provides wealth-constrained owners with funding at the outset of the relationship. Our findings support the statement that it is in the best interest of the organization involved in the program to offer a contract stipulating both (i) revenue sharing and (ii) a positive transfer at the outset of the relationship to owners characterized by low levels of wealth. However, Proposition 3 provides an argument for limiting the use of up-front payments to situations in which the interest rate is neither too low nor too high. Furthermore, our results also imply that an increase in the interest rate - depending on its initial level - may lead to an increase or to a decrease in the optimal additional payment.

\footnotetext{
${ }^{19}$ The discussion is consistent for the generic case of a natural resource.

${ }^{20}$ Regarding end-of-period payments, we refer to Munoz-Pina et al. (2008).

${ }^{21}$ For instance, in Brazil, farmers convert forests into cattle pasture land (Simonet et al. 2018).
} 


\section{Discussions}

\section{$5.1 \quad$ Limited liability}

Lemma 1 shows that a mix of incentives and funding may be optimal. We now show that this is incompatible with the classical notion of limited liability. We consider the same setting except that there is no monetary cost and we assume limited liability: the size of the financial transfer (in case money is transferred from the agent to the principal) is bounded from above. This is the version of limited liability that allows for a meaningful comparison: if one assumes that the agent simply cannot transfer money to the principal, then we rule out the possibility that funding would emerge endogenously. The principal's problem is thus as follows:

$$
\begin{gathered}
\underset{T, w, e}{\operatorname{Max}} E u^{F}=e(V-w)-T, \\
e \in \arg \max _{e \geq 0}\left\{e w+B+T-\psi \frac{e^{2}}{2}\right\}, \\
e w+B+T-\psi \frac{e^{2}}{2} \geq B, \\
T+B \geq-\bar{t}
\end{gathered}
$$

where $\bar{t}>0$ denotes the agent's liability limit. We obtain here:

Proposition 6: Under limited liability, it is not optimal for the principal to provide funding, that is: $T^{*} \leq 0$ is always satisfied.

This result highlights a notable difference with the classical notion of limited liability: this weak notion of limited liability is incompatible with the optimality of a mix of incentives and funding. The main difference is that the existence of a monetary cost makes the budget constraint binding at the optimal contract, and the transfer becomes an endogenous function of the agent's wealth: for sufficiently low budget levels, the optimal contract stipulates funding.

\subsection{What if the agent could borrow money from the principal?}

One way to relax the wealth constraint is to allow the agent to borrow from the principal. If this is possible ${ }^{22}$ what if the agent can repay the borrowed money after getting the bonus? We now explain that this additional option does not change the findings of our analysis. Let us consider that the agent can borrow money from the principal. The agent gets some fund at the outset of the project and may have to make a monetary transfer to the principal at the end of the project. This specific transfer may be conditional on the success or failure of the project. Let us denote $R_{s} \geq 0$ and $R_{f} \geq 0$ the amount of money the agent has to repay the principal ex-post if the project succeeds or fails, respectively. In this case, the principal has to take the agent's ex-post budget constraint into account. Then, it is easily checked that, redefining the bonus and the transfer from the principal to the agent as $w^{\prime} \equiv w-\left(R_{s}-R_{f}\right)$ and $T^{\prime} \equiv T-R_{f}$ and noticing that, as long as $w^{\prime} \geq 0$ is satisfied, which is valid under our assumptions (otherwise the agent

\footnotetext{
${ }^{22}$ In cases where institutions are weak, it may be quite difficult to ensure repayments of borrowed money, and this might severely restrict the option to borrow.
} 
would not exert effort), the more stringent budget constraint is the ex-post budget constraint when the project fails. The principal's objective function, the participation and the incentive constraints can also be rewritten using $w^{\prime}$ and $T^{\prime}$ instead of $w$ and $T$. It is then easily checked that the principal's problem is identical to the one introduced in the body of the article.

\subsection{Self-funding as an outside option}

So far the principal-agent relationship is exogenously given. This might be true if the relationship occurs within a given organization, or when the principal brings not only money but certain knowledge skills. The agent cannot initiate the project on his own: his outside option is equal to zero. By contrast, if the principal only brings money, then the relationship must be voluntarily chosen by the agent when he has positive initial wealth: indeed, he could rely on it to fund his project. Would the agent not be better off starting the project with just the effort afforded by his own wealth, instead of going to contract with a principal who will extract rents from him? If the agent relies on self-funding, his problem is

$$
\underset{e \geq 0}{\operatorname{Max}} E u^{A}=e V+B-\psi \frac{e^{2}}{2}-\theta \frac{e^{2}}{2}
$$

such that $B \geq \theta \frac{e^{2}}{2}$ holds. The solution is then given by $e^{S F}=\sqrt{\frac{2 B}{\theta}}$ when $B<\frac{\theta}{2}\left(\frac{V}{\psi+\theta}\right)^{2}$ is satisfied and by $e^{S F}=\frac{V}{\psi+\theta}=e^{F B}$ when $B \geq \frac{\theta}{2}\left(\frac{V}{\psi+\theta}\right)^{2}$ is satisfied. If self-funding constitutes the outside option, the participation constraint writes as follows:

$$
e w+T+B-\frac{\psi}{2} e^{2}-\frac{\theta}{2} e^{2} \geq \sqrt{\frac{2 B}{\theta}}\left[V-\frac{\psi}{2} \sqrt{\frac{2 B}{\theta}}\right]
$$

when $B<\frac{\theta}{2}\left(\frac{V}{\psi+\theta}\right)^{2}$ is satisfied, and

$$
e w+T+B-\frac{\psi}{2} e^{2}-\frac{\theta}{2} e^{2} \geq \frac{V^{2}}{2(\psi+\theta)}+B
$$

when $B \geq \frac{\theta}{2}\left(\frac{V}{\psi+\theta}\right)^{2}$ holds. We obtain the following result:

Proposition 7: The optimal contract $\left(w^{*}, T^{*}\right)$ is characterized as follows:

- For $B<\frac{\theta}{2}\left(\frac{V}{\psi}\right)^{2}\left[1-\frac{\sqrt{(2 \psi+3 \theta)^{2}-\psi(\psi+2 \theta)}}{2 \psi+3 \theta}\right]^{2} w^{*}=\frac{\psi+\theta}{2 \psi+3 \theta} V$ and $T^{*}=\frac{\theta}{2}\left(\frac{V}{2 \psi+3 \theta}\right)^{2}-B>0$

- For $B \in] \frac{\theta}{2}\left(\frac{V}{\psi}\right)^{2}\left[1-\frac{\sqrt{(2 \psi+3 \theta)^{2}-\psi(\psi+2 \theta)}}{2 \psi+3 \theta}\right]^{2}, \frac{\theta}{2}\left(\frac{V}{\psi+\theta}\right)^{2}[$ we have

$$
w^{*}=(\psi+\theta) \sqrt{\frac{2}{\psi+2 \theta}}\left(\sqrt{\frac{2 B}{\theta}}\left[V-\frac{\psi}{2} \sqrt{\frac{2 B}{\theta}}\right]\right)^{\frac{1}{2}} \text { and } T^{*}=\frac{\theta}{2} \frac{2}{\psi+2 \theta} \sqrt{\frac{2 B}{\theta}}\left[V-\frac{\psi}{2} \sqrt{\frac{2 B}{\theta}}\right]-B>0
$$

- Finally, for $B \geq \frac{\theta}{2}\left(\frac{V}{\psi+\theta}\right)^{2}$ we have $w^{*}=V$ and no transfer $T^{*}=0$

Thus funding occurs except when the agent's budget level is sufficiently high. This conclusion would be qualified if the principal's outside option would provide positive returns. Cases 
where self-funding would define the agent's outside option provides him with a notable bargaining advantage. The conclusions provided by the two conceptual settings considered here yield interesting insights, even more so if real-world cases actually lie in between these two settings. Indeed, both parties may have bargaining power in the contract definition process.

\section{Conclusion}

We analyze a contracting problem within a moral hazard setting where the agent's effort induces both monetary and non-monetary costs. We obtain several novel results with policy design implications, such as the optimality of outside financing for intermediate values of the monetary cost, or the non-monotonic effect of this cost on the incentive part of the optimal contract. This article opens up avenue for further research. Analyzing the case of a risk-averse agent (Holmstrom, 1979) constitutes a challenging next step of this research project: it would be interesting to assess how the usual trade-off between insurance and incentives would be affected in our setting ${ }^{23}$ Extending our analysis to a dynamic setting would be interesting as well.

\footnotetext{
${ }^{23}$ We thank a referee for this suggestion.
} 


\section{Appendix A: Proofs}

Proof of Proposition 1: Using Appendix B, we know that when $B \geq B_{2}(\theta)$, we have $T^{*} \leq 0$. Moreover, one can easily show that $B \geq B_{2}(\theta)$ is equivalent to $\theta \geq 0$ if $B \geq \frac{V^{2}}{2 \psi}$ and $\theta \geq \theta_{2} \equiv \frac{V^{2}-2 \psi B+V \sqrt{V^{2}-2 \psi B}}{2 B}$ if $B \leq \frac{V^{2}}{2 \psi}$.

Proof of Lemma 1: Let us first consider the case where $0 \leq B<B_{1}(\theta)$ is satisfied. We first prove the following property:

Property 1: If the agent's wealth level satisfies $0 \leq B<B_{1}$, the optimal contract is such that the bonus for success, $w^{*}$, is given by $w^{*}=\frac{\psi+\theta}{2 \psi+3 \theta} V$, the up-front payment from the principal to the agent is $T^{*}(B)=\frac{\theta}{2}\left(e^{*}\right)^{2}-B$, and the agent's effort level is $e^{*}=\frac{w^{*}}{\psi+\theta}=\frac{V}{2 \psi+3 \theta}<e^{F B}$.

Proof of Property 1: Assume that $e(V-w)-T \geq 0$ and $B<e^{*} w^{*}-\frac{\psi}{2}\left(e^{*}\right)^{2}$. We have that $e^{*}>0$, the principal's problem can then be written as

$$
\underset{e, w}{\operatorname{Max}} E u^{P}=e(V-w)-\frac{\theta}{2} e^{2}+B
$$

such that $e=\frac{w}{\psi+\theta}$ since $w^{*}>0$ is satisfied. We thus have $E u^{P}=\frac{w}{\psi+\theta}(V-w)-\frac{\theta}{2}\left(\frac{w}{\psi+\theta}\right)^{2}+B$. Let us show that $w^{*}<V$. If $w^{*} \geq V$ then the objective function $E u^{P}$ is decreasing in $w^{*}$, which is a contradiction. Hence, $w^{*}<V$. The principal's problem can then be rewritten as follows:

$$
\max _{w \in[0, V]} E u^{P}=\frac{w}{\psi+\theta}(V-w)+B-\frac{\theta}{2}\left(\frac{w}{\psi+\theta}\right)^{2} .
$$

Given that $E u^{P}$ is strictly concave and the maximum is not reached for 0 or $V$, the optimality condition yields $w^{*}=\frac{\psi+\theta}{2 \psi+3 \theta} V$. The principal's payoff is $E u^{P *}=\frac{w^{*}}{\psi+\theta}\left(V-w^{*}\right)-\frac{\theta}{2}\left(\frac{w^{*}}{\psi+\theta}\right)^{2}+$ $B=\frac{1}{2} \frac{V^{2}}{2 \psi+3 \theta}+B$, which is positive. The initial assumption that $B<e^{*} w^{*}-\frac{\psi}{2}\left(e^{*}\right)^{2}=$ $\frac{\psi+2 \theta}{2}\left(\frac{V}{2 \psi+3 \theta}\right)^{2}=B_{1}(\theta)$ is without loss of generality. First notice that, if $B>B_{1}(\theta)$, then we must have $w^{*}=V$, which is impossible. Second, let us show that an optimal contract cannot yield $B=e^{*} w^{*}-\frac{\psi}{2}\left(e^{*}\right)^{2}$. We have to show that the principal's expected surplus is larger when he chooses contract $\left(w^{*}, T^{*}\right)$ rather than contract $(\widetilde{w}, \widetilde{T})$ defined by $\frac{\widetilde{w}}{\psi+\theta} \widetilde{w}-\frac{\psi}{2}\left(\frac{\widetilde{w}}{\psi+\theta}\right)^{2}=B$ and $\widetilde{T}=\frac{\theta}{2}\left(\frac{\widetilde{w}}{\psi+\theta}\right)^{2}-B$. If the principal chooses contract $(\widetilde{w}, \widetilde{T})$ then its surplus is $\widetilde{e} V-\frac{\theta}{2}(\widetilde{e})^{2}$ with $\widetilde{e}=\frac{\widetilde{w}}{\psi+\theta}$. In the proof of Property 2, we show that the principal's surplus is continuous at $B=B_{1}$. It is then sufficient to prove that the derivative of $\widetilde{e} V-\frac{\theta}{2}(\widetilde{e})^{2}$ with respect to $B$ is strictly larger than the derivative of $E u^{P *}$ with respect to $B$ for all $B<B_{1}(\theta)$. Using $\widetilde{e} \widetilde{w}-\frac{\psi}{2}(\widetilde{e})^{2}=B$ and $\widetilde{e}=\frac{\widetilde{w}}{\psi+\theta}$, we obtain that the derivative of $\widetilde{e} V-\frac{\theta}{2}(\widetilde{e})^{2}$ with respect to $B$ is strictly larger than one if and only if $\frac{1}{\sqrt{2(\psi+2 \theta) B}}\left[V-\theta \sqrt{\frac{2 B}{\psi+2 \theta}}\right]>1$. This inequality holds for $B<B_{1}(\theta)$. Indeed, when $B=B_{1}(\theta)$, the left hand side term equals $1+\frac{\psi}{\psi+2 \theta}$. Moreover, the left hand side term is strictly decreasing in $B$. Hence, if $0 \leq B<B_{1}(\theta)$, then the principal proposes $w^{*}$ and $T^{*}=\frac{\theta}{2}\left(\frac{w^{*}}{\psi+\theta}\right)^{2}-B$. This concludes the proof of the first property.

Points (i) and (ii) follow from Property 1. Point (iv) follows from the proof of Property 1, as 
we know that $T^{*}=\frac{\theta}{2}\left(\frac{w^{*}}{\psi+\theta}\right)^{2}-B$, which is strictly positive if and only if $B<B_{0}(\theta)$. Now let us consider point (iii). From the proof of Property 1, we know that the participation constraint PC is not binding, thus we have $E u^{A *}>B$.

This concludes the proof of the first sub-case. Let us now consider that $B_{1}(\theta) \leq B<B_{2}(\theta)$ is satisfied. We first prove the following property:

Property 2: If the agent's wealth level is intermediate, $B_{1}(\theta) \leq B<B_{2}(\theta)$, the principal offers a contract such that both the budget and the participation constraints are binding. Moreover, the optimal effort level is $\widetilde{e}=\frac{\widetilde{w}}{\psi+\theta}$, the optimal transfer is $\widetilde{T}=\frac{\theta}{2}\left(\frac{\widetilde{w}}{\psi+\theta}\right)^{2}-B$, and the optimal bonus is characterized by equality $\frac{\widetilde{w}}{\psi+\theta} \widetilde{w}-\frac{\psi}{2}\left(\frac{\widetilde{w}}{\psi+\theta}\right)^{2}=B$, or $\tilde{w}=(\psi+\theta) \sqrt{\frac{2 B}{\psi+2 \theta}}$.

Proof of Property 2: We have $B_{1}(\theta) \leq B<B_{2}(\theta)$. Assume that $e(V-w)-T \geq 0$. If $B<\widetilde{e} \widetilde{w}-\frac{\psi}{2}(\widetilde{e})^{2}$, we know from the proof of Property 1 that we must have $B<B_{1}(\theta)$, which is a contradiction. If $B>\widetilde{e} \widetilde{w}-\frac{\psi}{2}(\widetilde{e})^{2}$, we know from the proof of Lemma 1 that we must have $B>B_{2}(\theta)$, which is a contradiction. Now assume that $\widetilde{e} \widetilde{w}-\frac{\psi}{2}(\widetilde{e})^{2}=B$. Using the incentive constraint, we have $\widetilde{e}=\frac{\widetilde{w}}{\psi+\theta}$. Using the budget constraint, we have $\widetilde{T}=\frac{\theta}{2}\left(\frac{\widetilde{w}}{\psi+\theta}\right)^{2}-B$. Moreover, in this case $\widetilde{w}$ is characterized by $\frac{\widetilde{w}}{\psi+\theta} \widetilde{w}-\frac{\psi}{2}\left(\frac{\widetilde{w}}{\psi+\theta}\right)^{2}=B$ and so $\tilde{w}=(\psi+\theta) \sqrt{\frac{2 B}{\psi+2 \theta}}$. It remains to show that the principal's surplus is non negative, i.e. $\widetilde{e}(V-\widetilde{w})-\widetilde{T} \geq 0$. Let us first show that the principal's payoff is continuous at $B=B_{1}(\theta)$. When $B=B_{1}(\theta)$, we have $\frac{\widetilde{w}}{\psi+\theta} \widetilde{w}-\frac{\psi}{2}\left(\frac{\widetilde{w}}{\psi+\theta}\right)^{2}=B_{1}(\theta)$, which is equivalent to $\frac{\widetilde{w}}{\psi+\theta} \widetilde{w}-\frac{\psi}{2}\left(\frac{\widetilde{w}}{\psi+\theta}\right)^{2}=\frac{w^{*}}{\psi+\theta} w^{*}-\frac{\psi}{2}\left(\frac{w^{*}}{\psi+\theta}\right)^{2}$. So we must have $w^{*}=\widetilde{w}$ and then $T^{*}=\widetilde{T}, e^{*}=\widetilde{e}$ and then the principal's payoff is continuous at $B=B_{1}(\theta)$. Now let us show that the principal's payoff is continuous at $B=B_{2}(\theta)$. When $B \rightarrow B_{2}(\theta)$, we have $\frac{\widetilde{w}}{\psi+\theta} \widetilde{w}-\frac{\psi}{2}\left(\frac{\widetilde{w}}{\psi+\theta}\right)^{2} \rightarrow B_{2}(\theta)$, or $\frac{\widetilde{w}}{\psi+\theta} \widetilde{w}-\frac{\psi}{2}\left(\frac{\widetilde{w}}{\psi+\theta}\right)^{2} \rightarrow \frac{V}{\psi+\theta} V-\frac{\psi}{2}\left(\frac{V}{\psi+\theta}\right)^{2}$. Notice that the derivative of $\frac{x}{\psi+\theta} x-\frac{\psi}{2}\left(\frac{x}{\psi+\theta}\right)^{2}$ with respect to $x$ is positive. Hence, when $B \rightarrow B_{2}(\theta)$, we have $\widetilde{w} \rightarrow V, \widetilde{e} \rightarrow e^{F B}, \widetilde{T} \rightarrow T^{F B}$ and then the principal's payoff is continuous at $B=B_{2}(\theta)$. Now, since $\widetilde{w}$ is strictly increasing in $B$ we have $w^{*} \leq \widetilde{w} \leq V$. Finally, let us show that the principal's payoff is strictly increasing in $B$. It can be written as $\frac{\widetilde{w}}{\psi+\theta} V-\frac{\theta}{2}\left(\frac{\widetilde{w}}{\psi+\theta}\right)^{2}$. The derivative of this expression with respect to $B$ is given by $\frac{d \widetilde{w}}{d B} \frac{V(\psi+\theta)-\theta \widetilde{w}}{(\psi+\theta)^{2}}$, which is positive (as $\frac{d \widetilde{w}}{d B}>0$ holds). Since the principal's surplus is strictly positive for $B=B_{1}(\theta)$, it is strictly positive for $B_{1}(\theta) \leq B<B_{2}(\theta)$. This concludes the proof of the second property.

The results related to the second sub-case follow from the proof of Property 2.

Proof of Proposition 4: To prove point (i), we note that $\frac{\partial T^{F B}}{\partial V}=-e^{F B}<0$ while $\frac{\partial W^{F B}}{\partial V}>0$ follows from the characterization of optimal incentives. Point (ii) is immediate. Point (iii) is immediate from Propositions 1 and 2.

Proof of Proposition 5: The result follows from the definition of both parties' surpluses.

Proof of Proposition 6: Since the principal's payoff decreases with the up-front payment, the principal may decrease the level of the up-front payment as long as the participation and bounded payment constraints are not binding. Thus, we have

$$
T^{*}=\max \left\{-\left[e w-\frac{\psi}{2} e^{2}\right],-B-\bar{t}\right\}
$$


The principal's problem can then be rewritten as follows

$$
\begin{gathered}
\underset{w, e}{\operatorname{Max} E u^{P}=e(V-w)-\max }\left\{-\left[e w-\frac{\psi}{2} e^{2}\right],-B-\bar{t}\right\}, \\
e \in \arg \max _{e \geq 0}\left\{e w-\frac{\psi}{2} e^{2}\right\} .
\end{gathered}
$$

Case $w^{*} \leq 0$ is non-optimal, and case where $e^{*}=0$ is not optimal either.

We have two cases to consider. First, if $-\left[e w-\frac{\psi}{2} e^{2}\right] \leq-B-\bar{t}$ then $T^{*}=-B-\bar{t}<0$ and the conclusion follows. We now consider that $-\left[e w-\frac{\psi}{2} e^{2}\right] \geq-B-\bar{t}$ holds, so $T^{*}=-\left[e w-\frac{\psi}{2} e^{2}\right]$ holds. The principal's problem is:

$$
\begin{gathered}
\underset{w, e}{\operatorname{Max}} E u^{P}=e V-\frac{\psi}{2} e^{2} \\
e \in \arg \max _{e \geq 0}\left\{e w-\frac{\psi}{2} e^{2}\right\} .
\end{gathered}
$$

and

$$
-\left[e w-\frac{\psi}{2} e^{2}\right] \geq-B-\bar{t}
$$

We obtain from (12) that $e^{*}=\frac{w}{\psi}$ holds. Then, condition 13 can be rewritten as $w \leq$ $\sqrt{2 \psi(B+\bar{t})}$. Finally, denoting $\lambda$ and $\mu$ the Lagrangian parameters associated to the principal's maximization program, we obtain the following optimality condition:

$$
\frac{V-w^{*}}{\psi}+\lambda-\mu=0
$$

We deduce easily that $\lambda=0$ as $w^{*}>0$ necessarily; moreover, if $\mu>0$ then $w^{*}=\sqrt{2 \psi(B+\bar{t})}$ and $T^{*}=-B-\bar{t}<0$ necessarily, which would conclude the proof. The last case to consider is the case $\lambda=0=\mu$ which yields $w^{*}=V$ and $T^{*}=-\frac{V^{2}}{2 \psi}<0$ holds. This concludes the proof.

Proof of Proposition 7: Let us first consider the case where $B<\frac{\theta}{2}\left(\frac{V}{\psi+\theta}\right)^{2}$ is satisfied. As in the proof of Proposition 1 it is deduced that the optimal transfer is $T=\frac{\theta}{2} e^{2}-\min \{B, B-$ $\left.\frac{\psi}{2} e^{2}+e w-\sqrt{\frac{2 B}{\theta}}\left[V-\frac{\psi}{2} \sqrt{\frac{2 B}{\theta}}\right]\right\}$ and then there are different cases to consider.

First, if $B<B-\frac{\psi}{2} e^{2}+e w-\sqrt{\frac{2 B}{\theta}}\left[V-\frac{\psi}{2} \sqrt{\frac{2 B}{\theta}}\right]$ holds at the optimal contract, then the proof of Proposition 1 yields $e^{*}=\frac{w^{*}}{\psi+\theta}$ with $w^{*}=\frac{\psi+\theta}{2 \psi+3 \theta} V$ due to the optimality condition derived from the principal's expected payoff $e^{*}\left(V-w^{*}\right)-\frac{\theta}{2}\left(e^{*}\right)^{2}+B$ and we obtain that $E u^{P}=\frac{1}{2} \frac{V^{2}}{2 \psi+3 \theta}+B>$ 0 is satisfied. But then we need to check that $B<B-\frac{\psi}{2}\left(e^{*}\right)^{2}+e^{*} w^{*}-\sqrt{\frac{2 B}{\theta}}\left[V-\frac{\psi}{2} \sqrt{\frac{2 B}{\theta}}\right]$ holds, that is,

$$
\sqrt{\frac{2}{\theta}} \sqrt{B} V-\frac{\psi}{\theta} B-\frac{\psi+2 \theta}{2}\left(\frac{V}{2 \psi+3 \theta}\right)^{2}<0
$$

Denoting $X:=\sqrt{B}$ this inequality can be rewritten as

$$
\sqrt{\frac{2}{\theta}} V X-\frac{\psi}{\theta} X^{2}-\frac{\psi+2 \theta}{2}\left(\frac{V}{2 \psi+3 \theta}\right)^{2}<0
$$


Solving for $X$, this is satisfied if and only if either $X<\underline{X}=\sqrt{\frac{\theta}{2}} \frac{V}{\psi}\left[1-\frac{\sqrt{(2 \psi+3 \theta)^{2}-\psi(\psi+2 \theta)}}{2 \psi+3 \theta}\right]$ or $X>\bar{X}=\sqrt{\frac{\theta}{2}} \frac{V}{\psi}\left[1+\frac{\sqrt{(2 \psi+3 \theta)^{2}-\psi(\psi+2 \theta)}}{2 \psi+3 \theta}\right]$ holds. Since $X<\sqrt{\frac{\theta}{2}} \frac{V}{\psi+\theta}$ necessarily holds, we deduce that $X>\bar{X}$ cannot hold as $\bar{X} \geq \sqrt{\frac{\theta}{2}} \frac{V}{\psi+\theta}$ and $\underline{X} \leq \sqrt{\frac{\theta}{2}} \frac{V}{\psi+\theta}$ ensures that we have $\sqrt{B}<\underline{X}$, which concludes the first sub-case.

Now consider the case where $B \in\left[\frac{\theta}{2}\left(\frac{V}{\psi}\right)^{2}\left[1-\frac{\sqrt{(2 \psi+3 \theta)^{2}-\psi(\psi+2 \theta)}}{2 \psi+3 \theta}\right]^{2}, \frac{\theta}{2}\left(\frac{V}{\psi+\theta}\right)^{2}[\right.$ holds. If now $B \geq B-\frac{\psi}{2} e^{2}+e w-\sqrt{\frac{2 B}{\theta}}\left[V-\frac{\psi}{2} \sqrt{\frac{2 B}{\theta}}\right]$ holds at the optimal contract, then the proof of Proposition 1 yields $e^{*}=\frac{w^{*}}{\psi+\theta}$ with $w^{*}=V$ due to the optimality condition derived from the principal's expected payoff $e^{*} V-\frac{\theta}{2}\left(e^{*}\right)^{2}-\frac{\psi}{2}\left(e^{*}\right)^{2}+B-\sqrt{\frac{2 B}{\theta}}\left[V-\frac{\psi}{2} \sqrt{\frac{2 B}{\theta}}\right]$ and we obtain that $E u^{P}=\frac{V^{2}}{2(\psi+\theta)}+\frac{\psi+\theta}{\theta} B-\sqrt{\frac{2 B}{\theta}} V>0$ holds. But then we need to check that $B \geq B-\frac{\psi}{2}\left(e^{*}\right)^{2}+$ $e^{*} w^{*}-\sqrt{\frac{2 B}{\theta}}\left[V-\frac{\psi}{2} \sqrt{\frac{2 B}{\theta}}\right]$ holds, that is,

$$
-\sqrt{\frac{2}{\theta}} \sqrt{B} V+\frac{\psi}{\theta} B+\frac{\psi+2 \theta}{2}\left(\frac{V}{\psi+\theta}\right)^{2} \leq 0
$$

Denoting $X:=\sqrt{B}$ this inequality can be rewritten as

$$
-\sqrt{\frac{2}{\theta}} V X+\frac{\psi}{\theta} X^{2}-\frac{\psi+2 \theta}{2}\left(\frac{V}{\psi+\theta}\right)^{2} \leq 0
$$

Solving for $X$, this is satisfied only if $X>\underline{X}=\sqrt{\frac{\theta}{2}} \frac{V}{\psi}\left[1-\frac{\theta}{\psi+\theta}\right]=\sqrt{\frac{\theta}{2}} \frac{V}{\psi+\theta}$. Since $X<\sqrt{\frac{\theta}{2}} \frac{V}{\psi+\theta}$ holds, we obtain a contradiction.

All together these conclusions imply that, when $B \in\left[\frac{\theta}{2}\left(\frac{V}{\psi}\right)^{2}\left[1-\frac{\sqrt{(2 \psi+3 \theta)^{2}-\psi(\psi+2 \theta)}}{2 \psi+3 \theta}\right]^{2}, \frac{\theta}{2}\left(\frac{V}{\psi+\theta}\right)^{2}[\right.$ holds, both the participation and the budget constraints bind. Thus $T^{*}=\frac{\theta}{2}\left(\frac{\tilde{w}}{\psi+\theta}\right)^{2}-B$ and $w^{*}$ satisfies:

$$
\frac{\psi+2 \theta}{2}\left(\frac{w^{*}}{\psi+\theta}\right)^{2}=\sqrt{\frac{2 B}{\theta}}\left[V-\frac{\psi}{2} \sqrt{\frac{2 B}{\theta}}\right]
$$

We obtain $w^{*}=(\psi+\theta) \sqrt{\frac{2}{\psi+2 \theta}}\left(\sqrt{\frac{2 B}{\theta}}\left[V-\frac{\psi}{2} \sqrt{\frac{2 B}{\theta}}\right]\right)^{\frac{1}{2}}$ and $T^{*}=\frac{\theta}{2} \frac{2}{\psi+2 \theta} \sqrt{\frac{2 B}{\theta}}\left[V-\frac{\psi}{2} \sqrt{\frac{2 B}{\theta}}\right]-$ $B$ is positive. Tedious algebra allows to check that the principal's expected payoff is non-negative. This concludes the proof of case $B<\frac{\theta}{2}\left(\frac{V}{\psi+\theta}\right)^{2}$.

Finally, when $B \geq \frac{\theta}{2}\left(\frac{V}{\psi+\theta}\right)^{2}$, the optimal transfer is $T^{*}=\frac{\theta}{2} e^{2}-\min \left\{B, e w-\frac{\psi}{2} e^{2}-\frac{V^{2}}{2(\psi+\theta)}\right\}$ and if $B \leq e w-\frac{\psi}{2} e^{2}-\frac{V^{2}}{2(\psi+\theta)}$ would be satisfied then $w^{*}=\frac{\psi+\theta}{2 \psi+3 \theta} V$ and $e^{*}=\frac{w^{*}}{\psi+\theta}$ but then it is easily checked that $B \leq e^{*} w^{*}-\frac{\psi}{2}\left(e^{*}\right)^{2}-\frac{V^{2}}{2(\psi+\theta)}$ is not satisfied, which is a contradiction. Thus $B>e w-\frac{\psi}{2} e^{2}-\frac{V^{2}}{2(\psi+\theta)}$ and $w^{*}=V$ due to the optimality condition derived from the principal's expected payoff $e^{*} V-\frac{\left(w^{*}\right)^{2}}{2(\psi+\theta)}-\frac{V^{2}}{2(\psi+\theta)}$ where $e^{*}=\frac{w^{*}}{\psi+\theta}$. Condition $B>e^{*} w^{*}-\frac{\psi}{2}\left(e^{*}\right)^{2}-\frac{V^{2}}{2(\psi+\theta)}$ is then equivalent to $B>\frac{\theta}{2}\left(\frac{V}{\psi+\theta}\right)^{2}$ which holds by assumption. The optimal transfer is $T^{*}=$ 
$\frac{\theta}{2}\left(\frac{V}{\psi+\theta}\right)^{2}-\left[\frac{V^{2}}{\psi+\theta}-\frac{\psi}{2}\left(\frac{V}{\psi+\theta}\right)^{2}-\frac{V^{2}}{2(\psi+\theta)}\right]=0$

\section{Appendix B: Link between problem $(\sqrt{\mathrm{P}})$ and problem $(\overline{\mathrm{P} 0})$.}

Let us focus on the incentive constraint $(\mathrm{IC} 0$ and forget the constraint $e \geq 0$ for now. The Lagrangian of this problem is:

$$
L=e w+B+T-\psi \frac{e^{2}}{2}-\theta \frac{e^{2}}{2}+\lambda\left(\sqrt{\frac{2(B+T)}{\theta}}-e\right) .
$$

The necessary conditions, which are also sufficient, are:

$$
\begin{aligned}
w-(\psi+\theta) e-\lambda & =0, \\
\lambda\left(\sqrt{\frac{2(B+T)}{\theta}}-e\right) & =0, \\
\lambda & \geq 0 .
\end{aligned}
$$

These conditions can be used in problem $\mathrm{P0}$ instead of $\sqrt{\mathrm{IC} 0}$. If $\lambda>0$ then $\sqrt{\frac{2(B+T)}{\theta}}=e<$ $\frac{w}{\psi+\theta}$ holds necessarily. Coming back to the principal's program the optimality conditions then yield that $(\mathrm{PC} 0)$ is necessarily binding. This in turn implies

$$
\sqrt{\frac{2(B+T)}{\theta}} w+T-\frac{\psi+\theta}{\theta}(B+T)=0
$$

so we obtain $w=\sqrt{\frac{\theta}{2(B+T)}} \frac{(\psi+\theta) B+\psi T}{\theta}$. There are two cases to consider here. If $(\psi+\theta)(B+$ $T)+\theta T \geq 0$, then $w<(\psi+\theta) \sqrt{\frac{2(B+T)}{\theta}}$ which can be satisfied only when $\lambda>0$ is not satisfied. Now, if $(\psi+\theta)(B+T)+\theta T<0$ then the principal's objective function is:

$$
E u^{P}=\sqrt{\frac{2(B+T)}{\theta}} V-\frac{\psi+\theta}{\theta} B-\frac{\psi+\theta}{\theta} T .
$$

It reaches its maximum value when $T=\frac{\theta}{2}\left(\frac{V}{\psi+\theta}\right)^{2}-B$. The incentive-part of the scheme is $w=\left(\frac{\psi+\theta}{V}\right) B+\frac{\psi}{2}\left(\frac{V}{\psi+\theta}\right)$, and using $(\psi+\theta)(B+T)+\theta T<0$ we obtain $B>\frac{\psi+2 \theta}{2}\left(\frac{V}{\psi+\theta}\right)^{2}$.

There is another candidate for the optimal contract in this case. Indeed, when $\lambda=0$ and the constraint holds, we know that the principal makes the agent residual claimant $(w=V)$ and ask him a lump sum transfer that is equal to the first best joint surplus $\left(T=-J^{F B}=-\frac{1}{2} \frac{V^{2}}{\psi+\theta}\right)$. Notice that the budget constraint thus holds only if $B \geq \frac{\psi+2 \theta}{2}\left(\frac{V}{\psi+\theta}\right)^{2}$.

These two options yield the same effort level and payoffs to both parties. If $B<\frac{\psi+2 \theta}{2}\left(\frac{V}{\psi+\theta}\right)^{2}$ we must have $\lambda=0$ and we thus conclude that problems $(\mathrm{P} 0)$ and $(\mathrm{P})$ yield the same solution in this case. 


\section{References}

Alix-Garcia, J. and H. Wolff (2014). Payment for ecosystem services from forests. Annual Review of Resource Economics 6, 361-380.

Arrow, K. (1963). Uncertainty and the welfare economics of medical care. American Economic Review 53(5), $941-973$.

Azam, J.-P. and J.-J. Laffont (2003). Contracting for aid. Journal of Development Economics $70(1), 25-58$.

Baron, D. (2007). Corporate social responsibility and social entrepreneurship. Journal of Economics and Management Strategy 16(3), 683 - 717.

Benoît, J.-P. and V. Krishna (2001). Multiple-object auctions with budget-constrained bidders. Review of Economic Studies 68(1), 155 - 179.

Bester, H. (1987). The role of collateral in credit markets with imperfect information. European Economic Review 31(4), 887 - 899.

Burkett, J. (2015). Endogenous budget constraints in auctions. Journal of Economic Theory 158(Part A), $1-20$.

Chassang, S. (2013). Calibrated incentive contracts. Econometrica 81(5), 1935 - 1971.

Che, Y.-K. and I. Gale (2000). The optimal mechanism for selling to a budget-constrained buyer. Journal of Economic Theory 92(2), 198 - 233.

Cordella, T. and G. Dell'Ariccia (2007). Budget support versus project aid: A theoretical appraisal. Economic Journal 117(523), 1260-1279.

Fagart, M.-C. and C. Fluet (2013). The first-order approach when the cost of effort is money. Journal of Mathematical Economics 49, $7-16$.

Heyes, A. and S. Martin (2016). Social labeling by competing ngos: A model with multiple issues and entry. Management Science 54(1), $151-166$.

Holmstrom, B. (1979). Moral hazard and observability. Bell Journal of Economics 10(1), 74-91.

Innes, R. (1990). Limited liability and incentive contracting with ex-ante action. Journal of Economic Theory 52, 45-67.

Jayachandran, S. (2013). Liquidity constraints and deforestation: The limitations of payments for ecosystem services. American Economic Review 103(3), 309-13.

Jelovac, I. and S. Kembou-Nzale (2020). Regulation and altruism. Journal of Public Economic Theory 22(1), 49-68.

Jewitt, I., O. Kadan, and J. Swinkels (2008). Moral hazard with bounded payments. Journal of Economic Theory 143(1), $59-82$.

Kawasaki, S. and J. McMillan (1987). The design of contracts: Evidence from japanese subcontracting. Journal of the Japanese and International Economies 1(3), $327-349$.

Kirkegaard, R. (2017). A unifying approach to incentive compatibility in moral hazard problems. Theoretical Economics 12, 25 - 51.

Laffont, J.-J. and M. S. Matoussi (1995). Moral hazard, financial constraints and sharecropping in el oulja. Review of Economic Studies 62(3), 381-399. 
Lewis, T. R. and D. E. M. Sappington (2000a). Contracting with wealth constrained agents. International Economic Review 41(3), 743-67.

Lewis, T. R. and D. E. M. Sappington (2000b). Motivating wealth constrained actors. American Economic Review 90(4), 944-960.

Lewis, T. R. and D. E. M. Sappington (2001). Optimal contracting with private knowledge of wealth and ability. Review of Economic Studies 68(1), 21-44.

Macho-Stadler, I. and D. Pérez-Castrillo (2018). Moral hazard: Base models and two extensions. In L. Corchon and M. Marini (Eds.), Handbook of Game Theory and Industrial Organization. Edward Elgar.

Mirrlees, J. (1976). The optimal structure of incentives and authority within an organization. Bell Journal of Economics 7(1), 105 - 131.

Munoz-Pina, C., A. Guevara, J. Torres, and J. Brana (2008). Paying for the hydrological services of mexico's forests: Analysis, negotiations and results. Ecological Economics 65(4), 725 - 736.

Ohlendorf, S. and P. Schmitz (2012). Repeated moral hazard and contracts with memory: The case of risk-neutrality. International Economic Review 53(2), 433 - 452.

Pauly, M. (1968). The economics of moral hazard: Comment. American Economic Review 58(3), $531-537$.

Poblete, J. and D. Spulber (2012). The form of incentive contracts: agency with moral hazard, risk neutrality, and limited liability. Rand Journal of Economics 43(2), 215 - 234.

Rogerson, W. (1985). The first-order approach to principal-agent problems. Econometrica 53(6), $1357-1367$.

Sappington, D. (1983). Limited liability contracts between principal and agent. Journal of Economic Theory 29, 1 - 21.

Simonet, G., J. Subervie, D. E. de Blas, M. Cromberg, and A. E. Duchelle (2018). Effectiveness of a redd+ project in reducing deforestation in the brazilian amazon. American Journal of Agricultural Economics 101(1), 211-229. 